\title{
Hierarchy and Up-Down Parallelism of Quark Mass Matrices
}

\author{
Jian-wei Mei and Zhi-zhong Xing \\ Institute of High Energy Physics, Chinese Academy of Sciences, \\ P.O. Box 918 (4), Beijing 100039, China * \\ (Electronic address: xingzz@mail.ihep.ac.cn)
}

\begin{abstract}
In view of the quark mass hierarchy and in the assumption of the up-down parallelism, we derive two phenomenologically-favored patterns of Hermitian quark mass matrices from the quark flavor mixing matrix. We compare one of them with two existing Ansätze proposed by Rosner and Worah and by Roberts et al, and find that only the latter is consistent with the present experimental data.
\end{abstract}

PACS number(s): 12.15.Ff, 12.10.Kt

*Mailing address 


\section{INTRODUCTION}

The masses and flavor mixing angles of six quarks are free parameters in the standard electroweak model, but their values can directly or indirectly be determined from a number of experiments [1]. The fact that three quark masses in each (up or down) sector perform a strong hierarchy is a big puzzle to particle physicists, so is the hierarchy of three quark mixing angles. Theoretical attempts to solve the puzzle, e.g., those starting from supersymmetric grand unification theories or from superstring theories [2], are encouraging but have not proved to be very successful. Phenomenologically, the common approach is to find out simple textures of quark mass matrices, from which a bridge between the hierarchy of quark mixing angles and that of quark masses can naturally be established [3]. The flavor symmetries hidden in such textures might finally provide us with useful hints towards the underlying dynamics responsible for the generation of quark masses and the origin of $C P$ violation.

The Cabibbo-Kobayashi-Maskawa (CKM) quark mixing matrix $V$ arises from the mismatch between the diagonalization of the up-type quark mass matrix $M_{\mathrm{U}}$ and that of the down-type quark mass matrix $M_{\mathrm{D}}$ [4]. Without loss of generality, one may arrange $M_{\mathrm{U}}$ and $M_{\mathrm{D}}$ to be Hermitian in the framework of the standard model or its extensions which have no flavor-changing right-handed currents [5]. There exist two reciprocal ways to explore possible relations between the hierarchy of $V$ and that of $M_{\mathrm{U}}$ and $M_{\mathrm{D}}$ :

(a) Taking account of a specific texture of the Hermitian quark mass matrix $M_{\mathrm{U}}$ or $M_{\mathrm{D}}$, which might result from a new flavor symmetry and its explicit breaking or rely on some plausible theoretical arguments, one may do the unitary transformation

$$
\begin{aligned}
O_{\mathrm{U}}^{\dagger} M_{\mathrm{U}} O_{\mathrm{U}} & =\left(\begin{array}{ccc}
\delta_{u} & 0 & 0 \\
0 & \delta_{c} & 0 \\
0 & 0 & \delta_{t}
\end{array}\right), \\
O_{\mathrm{D}}^{\dagger} M_{\mathrm{D}} O_{\mathrm{D}} & =\left(\begin{array}{ccc}
\delta_{d} & 0 & 0 \\
0 & \delta_{s} & 0 \\
0 & 0 & \delta_{b}
\end{array}\right),
\end{aligned}
$$

where $\delta_{i}$ stands for the mass eigenvalue of the $i$-quark. Then the CKM matrix $V$ is given as $V=O_{\mathrm{U}}^{\dagger} O_{\mathrm{D}}$, from which the quark mixing angles can be expressed in terms of the ratios of quark masses (and other unfixed parameters of $M_{\mathrm{U}}$ and $M_{\mathrm{D}}$ ). This normal approach has extensively been discussed in the literature [3].

(b) Taking account of the experimentally-allowed pattern of $V$ and making some proper assumptions to decompose $V$ into $V=O_{\mathrm{U}}^{\dagger} O_{\mathrm{D}}$ (e.g., the principle of "naturalness" disfavors any severe fine-tuning of or delicate cancellations between relevant parameters in the unitary transformation matrices $O_{\mathrm{U}}$ and $O_{\mathrm{D}}$ ), one may construct the Hermitian quark mass matrices $M_{\mathrm{U}}$ and $M_{\mathrm{D}}$ through

$$
\begin{aligned}
& M_{\mathrm{U}}=O_{\mathrm{U}}\left(\begin{array}{ccc}
\delta_{u} & 0 & 0 \\
0 & \delta_{c} & 0 \\
0 & 0 & \delta_{t}
\end{array}\right) O_{\mathrm{U}}^{\dagger}, \\
& M_{\mathrm{D}}=O_{\mathrm{D}}\left(\begin{array}{ccc}
\delta_{d} & 0 & 0 \\
0 & \delta_{s} & 0 \\
0 & 0 & \delta_{b}
\end{array}\right) O_{\mathrm{D}}^{\dagger} .
\end{aligned}
$$


This reverse approach has also attracted some attention in the literature [6].

In this Brief Report, we present a further study of the relationship between the CKM matrix and the quark mass matrices by following approach (b). Our main goal is to derive the phenomenologically-favored textures of Hermitian quark mass matrices from the CKM matrix. We are guided by the hierarchy of quark masses, the hierarchy of the CKM matrix elements and the afore-mentioned principle of naturalness. The only assumption that we need to make is the structural parallelism between up- and down-quark mass matrices. Such an empirical assumption should essentially be true, if two quark sectors are governed by the same dynamics. We arrive at two explicit textures of quark mass matrices, which were not obtained in Ref. [6]. One of them is particularly interesting, as its form is somehow similar to the Ansätze proposed by Rosner and Worah [7] and by Roberts et al [8]. A careful comparison shows that our pattern of quark mass matrices is compatible with the ansatz advocated in Ref. [8], while the Rosner-Worah pattern is not favored by the present experimental data.

\section{HIERARCHY AND UP-DOWN PARALLELISM}

The hierarchy of quark flavor mixing can clearly be seen from the Wolfenstein parametrization of the CKM matrix $V$ [9]:

$$
V \approx\left(\begin{array}{ccc}
1-\frac{1}{2} \lambda^{2}-\frac{1}{8} \lambda^{4} & \lambda & p \lambda^{3} \\
-\lambda & 1-\frac{1}{2} \lambda^{2}-\frac{1}{8}\left(1+4 A^{2}\right) \lambda^{4} & A \lambda^{2} \\
q \lambda^{3} & -A \lambda^{2}+\frac{1}{2}(2 q-A) \lambda^{4} & 1-\frac{1}{2} A^{2} \lambda^{4}
\end{array}\right)
$$

where $p \equiv A(\rho-i \eta), q \equiv A(1-\rho-i \eta)$, and the terms of or below $\mathcal{O}\left(\lambda^{5}\right)$ have been neglected. Current experimental data yield $\lambda \approx 0.22, A \approx 0.83, \rho \approx 0.17$ and $\eta \approx 0.36$ [10]. Thus we have

$$
\begin{aligned}
& |p|=A \sqrt{\rho^{2}+\eta^{2}} \approx 0.33 \\
& |q|=A \sqrt{(1-\rho)^{2}+\eta^{2}} \approx 0.75 .
\end{aligned}
$$

We find that $|p| /|q| \approx 2 \lambda$ holds and $\left|V_{u b}\right|=|p| \lambda^{3}$ is actually of $\mathcal{O}\left(\lambda^{4}\right)$.

To construct the Hermitian quark mass matrices $M_{\mathrm{U}}$ and $M_{\mathrm{D}}$ from $V$ through Eq. (2), the key point is to determine the unitary transformation matrices $O_{\mathrm{U}}$ and $O_{\mathrm{D}}$. As the physical quark masses $\left(m_{u}, m_{c}, m_{t}\right)$ or $\left(m_{d}, m_{s}, m_{b}\right)$ approximately perform a geometrical hierarchy at a common energy scale (e.g., the electroweak scale $\mu=M_{Z}[3]$ ),

$$
\begin{aligned}
& \frac{m_{u}}{m_{c}} \sim \frac{m_{c}}{m_{t}} \sim \zeta^{2}, \\
& \frac{m_{d}}{m_{s}} \sim \frac{m_{s}}{m_{b}} \sim \lambda^{2},
\end{aligned}
$$

where $\zeta$ is of $\mathcal{O}\left(\lambda^{2}\right)$ and can be defined as $\zeta \equiv \kappa \lambda^{2}$ with $\kappa \sim \mathcal{O}(1)$, we are allowed to expand the matrix elements of $M_{\mathrm{U}}$ or $M_{\mathrm{D}}$ in powers of $\zeta$ or $\lambda$. Provided two quark sectors are governed by the same dynamics, $M_{\mathrm{U}}$ and $M_{\mathrm{D}}$ are then expected to have approximately parallel structures characterized respectively by the perturbative parameters $\zeta$ and $\lambda$. This 
up-down parallelism would be exact, if the geometrical relations in Eq. (5) held exactly (i.e., $m_{u} / m_{c}=m_{c} / m_{t}=\zeta^{2}$ and $m_{d} / m_{s}=m_{s} / m_{b}=\lambda^{2}$ ). Note that the parallelism between $M_{\mathrm{U}}$ and $M_{\mathrm{D}}$ implies the parallelism between $O_{\mathrm{U}}$ and $O_{\mathrm{D}}$, whose textures can respectively be expanded in terms of $\zeta$ and $\lambda$. Since the CKM matrix $V=O_{\mathrm{U}}^{\dagger} O_{\mathrm{D}}$ deviates from the unity matrix only at the $\mathcal{O}(\lambda)$ level, both $O_{\mathrm{U}}$ and $O_{\mathrm{D}}$ must be close to the unity matrix in the spirit of naturalness. It is therefore instructive to expand $O_{\mathrm{U}}$ and $O_{\mathrm{D}}$ as follows:

$$
\begin{aligned}
& O_{\mathrm{U}}=I+\sum_{n=1}^{\infty}\left(Z_{n}^{\mathrm{U}} \zeta^{n}\right), \\
& O_{\mathrm{D}}=I+\sum_{n=1}^{\infty}\left(Z_{n}^{\mathrm{D}} \lambda^{n}\right),
\end{aligned}
$$

where $I$ stands for the unity matrix, and $Z_{n}^{\mathrm{U}}$ or $Z_{n}^{\mathrm{D}}$ (for $n=1,2,3, \cdots$ ) denotes the coefficient matrix of $\zeta^{n}$ or $\lambda^{n}$. While $Z_{n}^{\mathrm{U}} \sim Z_{n}^{\mathrm{D}}$ should in general hold, the simplest possibility is $Z_{n}^{\mathrm{U}}=Z_{n}^{\mathrm{D}}\left(\equiv Z_{n}\right)$, equivalent to the exact parallelism between $O_{\mathrm{U}}$ and $O_{\mathrm{D}}$. In this especially interesting case, the expressions of $Z_{1}, Z_{2}, Z_{3}$ and $Z_{4}$ can concretely be determined from Eqs. (3) and (6):

$$
\begin{aligned}
& Z_{1}=\left(\begin{array}{ccc}
0 & 1 & 0 \\
-1 & 0 & 0 \\
0 & 0 & 0
\end{array}\right), \\
& Z_{2}=\left(\begin{array}{ccc}
-\frac{1}{2} & \kappa & 0 \\
-\kappa & -\frac{1}{2} & A \\
0 & -A & 0
\end{array}\right), \\
& Z_{3}=\left(\begin{array}{ccc}
-\kappa & 0 & p \\
0 & -\kappa & 0 \\
q & 0 & 0
\end{array}\right), \\
& Z_{4}=\left(\begin{array}{ccc}
-\frac{1}{8}\left(1+4 \kappa^{2}\right) \\
\frac{1}{2} \kappa\left(1-2 \kappa^{2}\right) & -\frac{1}{8}\left(1+4 \kappa^{2}+4 A^{2}\right) & A \kappa^{2} \\
0 & -A \kappa^{2}+\frac{1}{2}\left(A-2 p^{*}\right) & -\frac{1}{2} A^{2}
\end{array}\right) .
\end{aligned}
$$

We see that the complex $C P$-violating phase enters $O_{\mathrm{U}}$ or $O_{\mathrm{D}}$ at the level of $\mathcal{O}\left(\zeta^{3}\right)$ or $\mathcal{O}\left(\lambda^{3}\right)$. As a straightfoward consequence of $\zeta \sim \mathcal{O}\left(\lambda^{2}\right)$, the contribution of $O_{\mathrm{D}}$ to $V$ is dominant over that of $O_{\mathrm{U}}$ to $V$.

With the help of Eqs. (6) and (7), we are now able to derive the hierarchical textures of $M_{\mathrm{U}}$ and $M_{\mathrm{D}}$ from Eq. (2). Note that the quark mass eigenvalues $\left(\delta_{u}, \delta_{c}, \delta_{t}\right)$ and $\left(\delta_{d}, \delta_{s}, \delta_{b}\right)$ in Eq. (2) may be either positive or negative. Without loss of generality, we take $\delta_{t}=m_{t}$ and $\delta_{b}=m_{b}$. The other four mass eigenvalues can be expressed, in view of Eq. (5), as follows:

$$
\begin{aligned}
\delta_{u} & =r_{u} \zeta^{4} m_{t}, \\
\delta_{c} & =r_{c} \zeta^{2} m_{t}
\end{aligned}
$$

and

$$
\begin{aligned}
& \delta_{d}=r_{d} \lambda^{4} m_{b}, \\
& \delta_{s}=r_{s} \lambda^{2} m_{b},
\end{aligned}
$$


where $\left|r_{u}\right| \sim\left|r_{c}\right| \sim\left|r_{d}\right| \sim\left|r_{s}\right| \sim \mathcal{O}(1)$ holds. Then we arrive at the results of $M_{\mathrm{U}}$ and $M_{\mathrm{D}}$ :

$$
\begin{gathered}
M_{\mathrm{U}} \approx m_{t}\left(\begin{array}{ccc}
\left(r_{u}+r_{c}\right) \zeta^{4} & \tilde{r}_{c} \zeta^{3} & p_{\zeta} \zeta^{3} \\
\tilde{r}_{c} \zeta^{3} & r_{c} \zeta^{2} & A \zeta^{2} \\
p_{\zeta}^{*} \zeta^{3} & A \zeta^{2} & 1
\end{array}\right), \\
M_{\mathrm{D}} \approx m_{b}\left(\begin{array}{ccc}
\left(r_{d}+r_{s}\right) \lambda^{4} & \tilde{r}_{s} \lambda^{3} & p_{\lambda} \lambda^{3} \\
\tilde{r}_{s} \lambda^{3} & r_{s} \lambda^{2} & A \lambda^{2} \\
p_{\lambda}^{*} \lambda^{3} & A \lambda^{2} & 1
\end{array}\right),
\end{gathered}
$$

where

$$
\begin{aligned}
& \tilde{r}_{c} \equiv r_{c}(1+\kappa \zeta), \\
& \tilde{r}_{s} \equiv r_{s}(1+\kappa \lambda)
\end{aligned}
$$

and

$$
\begin{aligned}
& p_{\zeta} \equiv p+\kappa A \zeta, \\
& p_{\lambda} \equiv p+\kappa A \lambda .
\end{aligned}
$$

We see that the parallelism between $M_{\mathrm{U}}$ and $M_{\mathrm{D}}$ in Eq. (10) is not exact (e.g., $p_{\zeta} \approx p \neq p_{\lambda}$ ), even though the exact parallelism between $O_{\mathrm{U}}$ and $O_{\mathrm{D}}$ has been assumed in our calculations.

Note that the sign uncertainties of quark mass eigenvalues may lead to two distinct textures of $M_{\mathrm{U}}$ and $M_{\mathrm{D}}$. If $r_{u}$ (or $r_{d}$ ) and $r_{c}$ (or $r_{s}$ ) have the same sign, the $(1,1)$ element of $M_{\mathrm{U}}\left(\right.$ or $\left.M_{\mathrm{D}}\right)$ amounts approximately to $2 r_{c} \zeta^{4}$ (or $\left.2 r_{s} \lambda^{4}\right)$. In this case, we obtain

$$
\begin{aligned}
& M_{\mathrm{U}} \approx m_{t}\left(\begin{array}{ccc}
2 r_{c} \zeta^{4} & \tilde{r}_{c} \zeta^{3} & p_{\zeta} \zeta^{3} \\
\tilde{r}_{c} \zeta^{3} & r_{c} \zeta^{2} & A \zeta^{2} \\
p_{\zeta}^{*} \zeta^{3} & A \zeta^{2} & 1
\end{array}\right), \\
& M_{\mathrm{D}} \approx m_{b}\left(\begin{array}{ccc}
2 r_{s} \lambda^{4} & \tilde{r}_{s} \lambda^{3} & p_{\lambda} \lambda^{3} \\
\tilde{r}_{s} \lambda^{3} & r_{s} \lambda^{2} & A \lambda^{2} \\
p_{\lambda}^{*} \lambda^{3} & A \lambda^{2} & 1
\end{array}\right) .
\end{aligned}
$$

If the signs of $r_{u}$ (or $r_{d}$ ) and $r_{c}$ (or $r_{s}$ ) are opposite to each other, however, a very significant cancellation must appear between them ${ }^{1}$. In this case, the $(1,1)$ elements of $M_{\mathrm{U}}$ and $M_{\mathrm{D}}$ are expected to be of or below $\mathcal{O}\left(\zeta^{5}\right)$ and $\mathcal{O}\left(\lambda^{5}\right)$, respectively. Then we are led to a somehow simpler texture of quark mass matrices:

$$
\begin{aligned}
& M_{\mathrm{U}} \approx m_{t}\left(\begin{array}{ccc}
0 & \tilde{r}_{c} \zeta^{3} & p_{\zeta} \zeta^{3} \\
\tilde{r}_{c} \zeta^{3} & r_{c} \zeta^{2} & A \zeta^{2} \\
p_{\zeta}^{*} \zeta^{3} & A \zeta^{2} & 1
\end{array}\right), \\
& M_{\mathrm{D}} \approx m_{b}\left(\begin{array}{ccc}
0 & \tilde{r}_{s} \lambda^{3} & p_{\lambda} \lambda^{3} \\
\tilde{r}_{s} \lambda^{3} & r_{s} \lambda^{2} & A \lambda^{2} \\
p_{\lambda}^{*} \lambda^{3} & A \lambda^{2} & 1
\end{array}\right) .
\end{aligned}
$$

\footnotetext{
${ }^{1}$ This cancellation does not involve two different quark sectors, thus it has no conflict with the principle of naturalness mentioned before.
} 
The textures of Hermitian quark mass matrices in Eqs. (13) and (14) result naturally from the quark mass hierarchy and the up-down parallelism. Therefore they can be regarded as two promising candidates for the "true" quark mass matrices in an underlying effective theory of quark mass generation at low energies.

\section{COMPARISON WITH TWO EXISTING ANSÄTZE}

Now let us compare the texture of quark mass matrices obtained in Eq. (14) with two existing Ansätze proposed by Rosner and Worah (RW) in Ref. [7] and by Roberts, Romanino, Ross and Velasco-Sevilla (RRRV) in Ref. [8]. Note that quark mass matrices of both RW and RRRV forms are symmetric instead of Hermitian. Their consequences on quark flavor mixing and $C P$ violation are essentially unchanged, however, if the Hermiticity is imposed on them [8]. For this reason, we consider the Hermitian versions of the RW and RRRV Ansätze and compare them with Eq. (14).

The RW ansatz of quark mass matrices is based on a composite model of spin-1/2 particles [7] and its Hermitian form can be written as

$$
\begin{gathered}
\mathcal{M}_{\mathrm{U}}=\left(\begin{array}{ccc}
0 & \sqrt{2} \alpha_{\mathrm{U}} & \alpha_{\mathrm{U}} \\
\sqrt{2} \alpha_{\mathrm{U}}^{*} & \beta_{\mathrm{U}} & \sqrt{2} \beta_{\mathrm{U}} \\
\alpha_{\mathrm{U}}^{*} & \sqrt{2} \beta_{\mathrm{U}} & \gamma_{\mathrm{U}}
\end{array}\right), \\
\mathcal{M}_{\mathrm{D}}=\left(\begin{array}{ccc}
0 & \sqrt{2} \alpha_{\mathrm{D}} & \alpha_{\mathrm{D}} \\
\sqrt{2} \alpha_{\mathrm{D}}^{*} & \beta_{\mathrm{D}} & \sqrt{2} \beta_{\mathrm{D}} \\
\alpha_{\mathrm{D}}^{*} & \sqrt{2} \beta_{\mathrm{D}} & \gamma_{\mathrm{D}}
\end{array}\right),
\end{gathered}
$$

where $\alpha_{\mathrm{U}}$ and $\alpha_{\mathrm{D}}$ are complex parameters so as to accommodate the observed effects of $C P$ violation in the quark sector [1]. Comparing between Eqs. (14) and (15), we find that the latter could basically be reproduced from the former, if the following conditions were satisfied:

$$
\begin{aligned}
& \frac{\left|\tilde{r}_{c}\right|}{\left|p_{\zeta}\right|} \approx \frac{A}{\left|r_{c}\right|} \approx \sqrt{2}, \\
& \frac{\left|\tilde{r}_{s}\right|}{\left|p_{\lambda}\right|} \approx \frac{A}{\left|r_{s}\right|} \approx \sqrt{2} .
\end{aligned}
$$

In view of Eqs. (8) and (9) as well as Eqs. (11) and (12), we immediately realize that the relations in Eq. (16) are impossible to hold. Hence the RW texture is actually incompatible with ours given in Eq. (14). Does this incompatibility imply the disagreement between the RW ansatz and current experimental data? We find that the answer is affirmative. Since the $(1,2)$ and $(1,3)$ elements of $\mathcal{M}_{\mathrm{U}}$ or $\mathcal{M}_{\mathrm{D}}$ in Eq. (15) are comparable in magnitude, we are led to the prediction

$$
\left|V_{t d}\right| \approx\left|V_{u b}\right| \sim \frac{1}{\sqrt{2}} \lambda^{3}
$$

in the leading-order approximation. Such a result is obviously inconsistent with the present experimental data, which require $\left|V_{u b}\right| \sim \mathcal{O}\left(\lambda^{4}\right)$ and $\left|V_{u b} / V_{t d}\right| \approx|p| /|q| \approx 2 \lambda$. Thus the 
RW texture of quark mass matrices, no matter whether it is Hermitian or symmetric, is no longer favored in phenomenology.

The Hermitian RRRV ansatz of quark mass matrices [8] takes the form ${ }^{2}$

$$
\begin{aligned}
& \mathcal{M}_{\mathrm{U}}=m_{t}\left(\begin{array}{ccc}
0 & b_{\mathrm{U}} \epsilon_{\mathrm{U}}^{3} & c_{\mathrm{U}} \epsilon_{\mathrm{U}}^{4} \\
b_{\mathrm{U}}^{*} \epsilon_{\mathrm{U}}^{3} & \epsilon_{\mathrm{U}}^{2} & a_{\mathrm{U}} \epsilon_{\mathrm{U}}^{2} \\
c_{\mathrm{U}}^{*} \epsilon_{\mathrm{U}}^{4} & a_{\mathrm{U}}^{*} \epsilon_{\mathrm{U}}^{2} & 1
\end{array}\right), \\
& \mathcal{M}_{\mathrm{D}}=m_{b}\left(\begin{array}{ccc}
0 & b_{\mathrm{D}} \epsilon_{\mathrm{D}}^{3} & c_{\mathrm{D}} \epsilon_{\mathrm{D}}^{4} \\
b_{\mathrm{D}}^{*} \epsilon_{\mathrm{D}}^{3} & \epsilon_{\mathrm{D}}^{2} & a_{\mathrm{D}} \epsilon_{\mathrm{D}}^{2} \\
c_{\mathrm{D}}^{*} \epsilon_{\mathrm{D}}^{4} & a_{\mathrm{D}}^{*} \epsilon_{\mathrm{D}}^{2} & 1
\end{array}\right),
\end{aligned}
$$

where $\epsilon_{\mathrm{U}} \approx \sqrt{m_{c} / m_{t}}$ and $\epsilon_{\mathrm{D}} \approx \sqrt{m_{s} / m_{b}}$ stand respectively for the expansion parameters of up- and down-quark sectors, and the remaining parameters $\left(a_{\mathrm{U}}, b_{\mathrm{U}}, c_{\mathrm{U}}\right)$ and $\left(a_{\mathrm{D}}, b_{\mathrm{D}}, c_{\mathrm{D}}\right)$ are all of $\mathcal{O}(1)$ in magnitude. From Eqs. (8) and (9), we obtain the relation between $\epsilon_{\mathrm{U}}$ (or $\left.\epsilon_{\mathrm{D}}\right)$ and $\zeta($ or $\lambda)$ as follows:

$$
\begin{aligned}
& \epsilon_{\mathrm{U}} \approx \zeta \sqrt{\left|r_{c}\right|}, \\
& \epsilon_{\mathrm{D}} \approx \lambda \sqrt{\left|r_{s}\right|} .
\end{aligned}
$$

In addition, we find

$$
\begin{aligned}
\left|a_{\mathrm{U}}\right| & \approx \frac{A}{\left|r_{c}\right|}, \\
\left|b_{\mathrm{U}}\right| & \approx \frac{1+\kappa \zeta}{\sqrt{\left|r_{c}\right|}}, \\
\left|c_{\mathrm{U}}\right| & \approx \frac{\left|p_{\zeta}\right|}{\zeta\left|r_{c}\right|^{2}}
\end{aligned}
$$

and

$$
\begin{aligned}
& \left|a_{\mathrm{D}}\right| \approx \frac{A}{\left|r_{s}\right|}, \\
& \left|b_{\mathrm{D}}\right| \approx \frac{1+\kappa \lambda}{\sqrt{\left|r_{s}\right|}}, \\
& \left|c_{\mathrm{D}}\right| \approx \frac{\left|p_{\lambda}\right|}{\lambda\left|r_{s}\right|^{2}} .
\end{aligned}
$$

It is obvious that the moduli of $\left(a_{\mathrm{U}}, b_{\mathrm{U}}, c_{\mathrm{U}}\right)$ and $\left(a_{\mathrm{D}}, b_{\mathrm{D}}, c_{\mathrm{D}}\right)$ are all of $\mathcal{O}(1)$, consistent with the assumption made in Ref. [8] ${ }^{3}$. Hence the RRRV ansatz is compatible with our texture of

\footnotetext{
${ }^{2}$ This texture is indeed similar to the one discussed by Branco et al in Ref. [11].

${ }^{3}$ Note that $\left|c_{\mathrm{U}}\right| \sim \lambda / \zeta \sim 1 / \lambda$ may be around 4.5 , but it has little impact on the global fit of the RRRV ansatz to current experimental data [8].
} 
quark mass matrices given in Eq. (14) and favored by current experimental data on quark flavor mixing.

It is worth remarking that a simplification of the RRRV ansatz to the following four-zero texture

$$
\begin{aligned}
& \mathcal{M}_{\mathrm{U}}=m_{t}\left(\begin{array}{ccc}
0 & b_{\mathrm{U}} \epsilon_{\mathrm{U}}^{3} & 0 \\
b_{\mathrm{U}}^{*} \epsilon_{\mathrm{U}}^{3} & \epsilon_{\mathrm{U}}^{2} & a_{\mathrm{U}} \epsilon_{\mathrm{U}}^{2} \\
0 & a_{\mathrm{U}}^{*} \epsilon_{\mathrm{U}}^{2} & 1
\end{array}\right), \\
& \mathcal{M}_{\mathrm{D}}=m_{b}\left(\begin{array}{ccc}
0 & b_{\mathrm{D}} \epsilon_{\mathrm{D}}^{3} & 0 \\
b_{\mathrm{D}}^{*} \epsilon_{\mathrm{D}}^{3} & \epsilon_{\mathrm{D}}^{2} & a_{\mathrm{D}} \epsilon_{\mathrm{D}}^{2} \\
0 & a_{\mathrm{D}}^{*} \epsilon_{\mathrm{D}}^{2} & 1
\end{array}\right)
\end{aligned}
$$

would give rise to the prediction $\left|V_{u b}\right| /\left|V_{c b}\right| \approx \sqrt{m_{u} / m_{c}} \leq 0.06$ [12] for reasonable values of $m_{u}$ and $m_{c}$ [13], which is difficult to agree with the present experimental result $\left|V_{u b} / V_{c b}\right|_{\text {ex }} \approx$ 0.09 [1]. Such a discrepancy implies that the interesting four-zero pattern of quark mass matrices in Eq. (22) might no longer be favored ${ }^{4}$.

Comparing Eq. (18) with Eqs. (15) and (22), we see that the $(1,3)$ elements of $\mathcal{M}_{\mathrm{U}}$ and $\mathcal{M}_{\mathrm{D}}$ should be neither larger than or comparable with their neighboring $(1,2)$ elements nor vanishing or negligibly small. This observation is particularly true for $M_{\mathrm{D}}$, which contributes dominantly to the CKM matrix $V$.

\section{SUMMARY}

We have derived two phenomenologically-favored textures of Hermitian quark mass matrices from the CKM matrix. Our starting points of view include the hierarchy of quark masses, the hierarchy of the CKM matrix elements and the principle of naturalness. The main assumption that we have made is the structural parallelism between up- and downquark mass matrices, which is expected to be true if two quark sectors are governed by the same dynamics.

We have compared one of the obtained textures with two existing Ansätze of quark mass matrices, proposed by Rosner and Worah and by Roberts et al. It turns out that the Rosner-Worah ansatz is no more favored in phenomenology, while the ansatz of Roberts et $a l$ is in good agreement with current experimental data.

We hope that our results for the structures of quark mass matrices may serve as useful guides to model building, from which some deeper understanding of quark masses, flavor mixing and $C P$ violation can finally be achieved.

\section{ACKNOWLEDGMENTS}

This work was supported in part by the National Natural Science Foundation of China.

\footnotetext{
${ }^{4}$ Note, however, that the general four-zero texture of Hermitian quark mass matrices can still be in good agreement with current experimental data. See Ref. [14] for detailed discussions.
} 


\section{REFERENCES}

[1] Particle Data Group, K. Hagiwara et al., Phys. Rev. D 66, 010001 (2002).

[2] See, e.g., S. Dimopoulos, L.J. Hall, and S. Raby, Phys. Rev. Lett. 68, 1984 (1992); M. Leurer, Y. Nir, and N. Seiberg, Nucl. Phys. B 398, 319 (1993); L.E. Ibáñez and G.G. Ross, Phys. Lett. B 332, 100 (1994); K.S. Babu and R.N. Mohapatra, Phys. Rev. Lett. 74, 2418 (1995); T. Kobayashi and Z.Z. Xing, Mod. Phys. Lett. A 12, 561 (1997); Int. J. Mod. Phys. A 13, 2201 (1998); K. Matsuda, T. Fukuyama, and H. Nishiura, Phys. Rev. D 61, 053001 (2000); D. Cremades, L.E. Ibáñez, and F. Marchesano, hep-ph/0212064; M. Bando and M. Obara, hep-ph/0302034; and references therein.

[3] For a recent review with extensive references, see: H. Fritzsch and Z.Z. Xing, Prog. Part. Nucl. Phys. 45, 1 (2000).

[4] N. Cabibbo, Phys. Rev. Lett. 10, 531 (1963); M. Kobayashi and T. Maskawa, Prog. Theor. Phys. 49, 652 (1973).

[5] H. Fritzsch and Z.Z. Xing, Nucl. Phys. B 556, 49 (1999); and references therein.

[6] See, e.g., P.M. Fishbane and P.Q. Hung, Phys. Rev. D 45, 293 (1992); P. Ramond, R.G. Roberts, and G.G. Ross, Nucl. Phys. B 406, 19 (1993); R.D. Peccei and K. Wang, Phys. Rev. D 53, 2712 (1996).

[7] J.L. Rosner and M. Worah, Phys. Rev. D 46, 1131 (1992).

[8] R.G. Roberts, A. Romanino, G.G. Ross, and L. Velasco-Sevilla, Nucl. Phys. B 615, 358 (2001).

[9] L. Wolfenstein, Phys. Rev. Lett. 51, 1945 (1983); A.J. Buras, M.E. Lautenbacher, and G. Ostermaier, Phys. Rev. D 50, 3433 (1994); Z.Z. Xing, Phys. Rev. D 51, 3958 (1995).

[10] A.J. Buras, hep-ph/0210291; and references therein.

[11] G.C. Branco, D. Emmanuel-Costa, and R. Gonzalez Felipe, Phys. Lett. B 483, 87 (2000).

[12] See, e.g., D.Du and Z.Z. Xing, Phys. Rev. D 48, 2349 (1993); L.J. Hall and A. Rasin, Phys. Lett. B 315, 164 (1993); H. Fritzsch and Z.Z. Xing, Phys. Lett. B 353, 114 (1995); Phys. Lett. B 413, 396 (1997).

[13] J. Gasser and H. Leutwyler, Phys. Rep. C 87, 77 (1982); H. Leutwyler, Phys. Lett. B 378, 313 (1996).

[14] H. Fritzsch and Z.Z. Xing, Phys. Lett. B 555, 63 (2003); hep-ph/0212195. 